pregnancies and their sequelae were followed up in medical oncology. The data was collected from out patient \& in patient tickets \& admission registrars and was analysed by descriptive statistics.

Results Most cases were seen amongst the second gravida 40\%, Hindus 53.3\%, low socio-economic strata 72\%.Predominant Blood group was B 53\%. Hemoglobin below $10 \mathrm{mg} / \mathrm{dl}$ was seen in $94 \%$. $21.33 \%$ of patients had haemoglobing was below $6 \mathrm{gm} / \mathrm{dl}$.Most of the patients of Hydatiform Mole $(50 \%)$ were diagnosed within a period of ammenorhea of $8-$ 12 weeks with $70 \%$ of cases diagnosed with amenorrhoea of less than 16 weeks. $18 \%$ of patients were diagnosed after a period of amenorrhoea of greater than 20 weeks. The most common presenting symptom in cases of Hydatiform Mole was Bleeding per vagina $74 \%$. Features of Hyperthyroidism \& respiratory distress were seen in $5 \%$ of patients. The most common signs were pallor $65 \%$, pre-eclampsia were seen in $17.33 \% \%$ of patients.

Suction \& Evacuation 58.66\%\% with Oxytocin infusion was the predominant mode of management in cases of Hydatiform Mole. Ligation was done in one patient considering the risk of repeat molar pregnancy in future conception.

Modes of diagnosis were clinical (74\%), \& USG in $68 \%$.

Persistent Gestational Trophoblastic Disease and Choriocarcinoma were diagnosed during follow up by symptoms of irregular bleeding $\mathrm{P} / \mathrm{V}$, elevated beta $\mathrm{HCG}$ titre and abnormal USG pelvis and chest X-Ray.

Chemotherapy was the predominant mode of treatment of GTT. hysterectomy was done in 2 patients of Invasive mole.

Single agent chemotherapy with Methotrexate in 20 patients $83.33 \%$ i.e low risk GTT. EMA-Co regimen was the preferred multiagent chemotherapy used in 4 patients $18 \%$ (upfront) and in 2 patients progressing on methotrexate, surgery in 1patient not responding to EMACO or EMA-EP.

Toxicity of chemotherapy was predominantly, Nausea \& vomiting (38.89\%) mucousitis (27.78\%).Hepatotoxicity and infection was seen in $11.11 \%$ of patients. Grade3/Grade4 toxcity was nil.

Conclusion Though the proportion of molar pregnancies \& gestational trophoblastic neoplasia is not much in comparison to the heavy attendees in the gynaecology and obstetrics opd but they represent a highly curable one with minimally intense chemotherapy thus avoiding unnecessary hospital stay due to chemotoxicity.

Disclosure I do not have any conflict of interestwith any person or organization.

\section{NEW CHALLENGES IN THE MANAGEMENT AND FOLLOW-UP OF MOLAR PREGNANCY}

Anca Copos, Diana Mocuta, Romina Cuc, Cristina Aur. County Emergency Clinical Hospital of Oradea; University of Oradea, Faculty of Medicine; Obstetrics - Gynecology

\subsection{6/ijgc-2020-ESG0.174}

Introduction/Background Hydatidiform mole (HM) is the premalignant form of gestational trophoblastic neoplasia. This entity is of clinical and epidemiological interest because of its potential for significant consequences for women's reproductive health.

Methodology This is a retrospective study of all cases of molar pregnancy managed in County Emergency Clinical Hospital of Oradea from 1st January 2019 to 30th August 2020.
The details of maternal characteristics, clinical presentation, tumor type and management were studied.

Results We diagnosed 17 cases of molar pregnancy during this period of 20 months and wemanaged 5364 deliveries. We also recorded 614 miscarriages and histopathological exams performed. The mean age of the patients with $\mathrm{HM}$ was 27,17 years old, with the highest incidence in patients between 1520 years $(29,41 \%)$. From allthe cases of HM, 94,12\% were diagnosed in first trimester of pregnancy and we had only one case in second trimester pregnancy. Amenorrhea followed by vaginal bleeding was the common symptom in 14 cases $(82,3 \%)$. A number of 12 patients were admitted because of exaggerated forms of hyperemesis gravidarum. The ultrasound exam showed the size of the uterus larger than the amenorrhea and ovarian lutein cysts were present in almost half of cases. All the patients have had higher then normal values of HCG. In our department all the cases were managed with dilation, suctionand mild curettage when necessary, except one case, finalized with hysterectomy, because of the molar type and the patient's age. Histopathological exam was performed in all cases. In 11 cases $(64,7 \%)$ partial hydatidiform mole was diagnosed and in 6 cases complete HM (25,3\%). A serial determination of HCG until normal values was always recommended, but we could not do the correct monitoring up to 6-12 months in 7 cases, related to the migration of the population in the region.

Conclusion Molar pregnancy has remained an important cause of maternal morbidity and mortality. There is need for early diagnosis, for proper treatment and follow-up of this condition. Due to the frequent use of ultrasound scanning, the diagnosis of hydatidiform mole could be made early in pregnancy. If hydatidiform mole is suspected, the quantitative estimation of serum level ofHCG should be done. After an appropriate treatment, it is always necessary to follow-up the patient and in present this is a new challenge because the population migration due to new socio-economic conditions and modern life.

Disclosures I have nothing to disclose.

\section{METASTATIC POSTMOLAR CHORIOCARCINOMA OF THE SKIN}

Michelle Lureineil Dajao, Sherry Joahne Villariasa. Cebu Velez General Hospital; Obstetrics and Gynecology

\subsection{6/ijgc-2020-ESGO.175}

Introduction/Background Gestational choriocarcinoma is a malignant tumor arising from trophoblastic cells with the lung and the vagina as its common sites of metastasis. Skin metastasis is known to be extremely unusual. 1 This paper outlines the case of a 45-year-old multigravida who manifested with occasional nonproductive cough; multiple cutaneous lesions in left flank, right triceps area, upper back, and infraumbilical areas associated with neurologic symptoms, two years after undergoing hysterectomy for a molar pregnancy. Skin biopsy of the left flank masses showed metastatic gestational choriocarcinoma; and she had elevated B-hCG (309,245 mIU/mL), and lung, brain, liver, and right adrenal metastases on imaging studies. She achieved remission after treatment with Etoposide Cisplatin induction chemotherapy, high-dose EMACO with concurrent whole brain irradiation, and ten cycles of EMACO. 

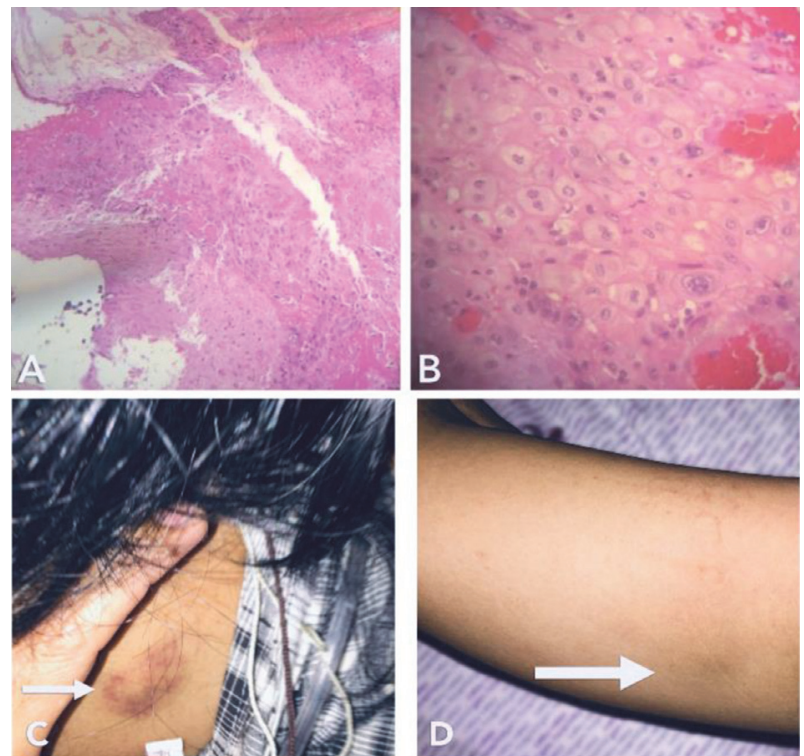

Abstract 434 Figure 1 Low power view of the skin biopsy of the patient's left flank mass - H\&E x 40 (A). High power view showing two cell populations of mononuclear cytotrophoblasts and multinucleated syncytiotrophoblasts in the tumor - H\&E x 100 (B). Skin metastasis in the upper back (C) and in the inner, medial portion of the right triceps (D).
Seven months after treatment, she remains alive and well, with ongoing regular follow-ups. The importance of keeping a high index of suspicion in patients with a prior molar pregnancy who only have clinical presentations referable to metastatic sites to avoid delay in the diagnosis and treatment; as well as the curability of widespread disease with aggressive combined treatment modalities, is emphasized herein (figure 1 and 2).

Methodology NA

Results NA

Conclusion Cutaneous metastases in gestational CC is infrequent and one of its diverse atypical clinical manifestations that has the potential to delay diagnosis and affect the clinical outcome. It is also associated with disseminated disease. Nevertheless, remission through aggressive multi-modal therapeutic strategies like Etoposide-Cisplatin induction chemotherapy, high-dose EMACO with concurrent whole brain irradiation, and regular EMACO is still possible for Stage IV multi-metastatic gestational CC patients who have late presentations and already have advanced disease, as documented in the index case. Prompt identification and vigorous treatment as keys to ensure better prognosis in gestational CC is stressed.

Disclosures N/A

\begin{tabular}{|c|c|c|c|c|c|c|c|c|c|}
\hline & 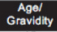 & 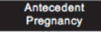 & & 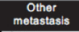 & & & & & \\
\hline 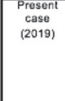 & 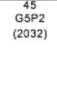 & 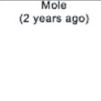 & 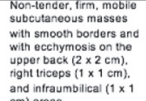 & 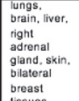 & & $\begin{array}{l}68.645 \\
\text { mulumL }\end{array}$ & 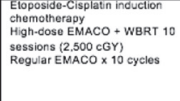 & & \\
\hline 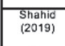 & 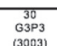 & (11/2years ago) & 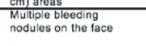 & & Crororocartchoma & $\begin{array}{l}31.50 \\
\text { mivum }\end{array}$ & Not mentitoned & None & 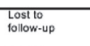 \\
\hline$(2015)$ & & $\begin{array}{l}\text { (42 yearts ago) } \\
\text { (e) }\end{array}$ & Two scalp masses & 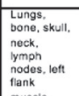 & Noldone & $3,17710 \mathrm{Un}$ & 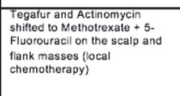 & None & 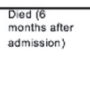 \\
\hline $\begin{array}{c}\text { chol } \\
\text { (2015) }\end{array}$ & ${ }^{30}$ & $\begin{array}{l}\text { (33 months ago) } \\
\text { (3) }\end{array}$ & 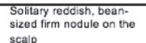 & Lungs & Croforcarticinoma & $\begin{array}{ll}275.673 \\
\text { milumL }\end{array}$ & Chemothorapy (not spectifod) & None & Not ment \\
\hline $\begin{array}{l}\text { Brotoks } \\
(2014)\end{array}$ & $\begin{array}{l}\text { ind day } \\
\text { old intant }\end{array}$ & & 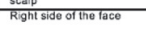 & Lungs & Choriocat & High & BEP & 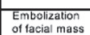 & Remissior \\
\hline 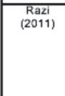 & $\begin{array}{l}47 \\
\begin{array}{l}443 \\
(3013)\end{array}\end{array}$ & 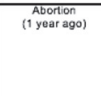 & 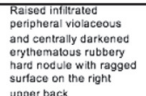 & Lungs & & $\begin{array}{l}\substack{\text { Nol } \\
\text { mentioned }} \\
\text { mate }\end{array}$ & 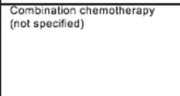 & & Nolme \\
\hline 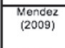 & $\begin{array}{l}232 \\
6392 \\
(20011)\end{array}$ & 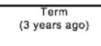 & 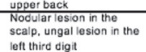 & 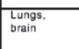 & 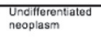 & $\Gamma 200.000$ & EMACO + WBRT $\times 4$ cyclos & None & $\begin{array}{l}\text { Deved } \\
\text { months atter } \\
\text { diagnosis }\end{array}$ \\
\hline $\begin{array}{l}\text { Cintrsta } \\
\text { (2008) }\end{array}$ & & 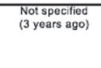 & 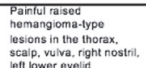 & Lung & Not dona & $\begin{array}{l}\substack{\text { Not } \\
\text { mentioned }} \\
\text { mate }\end{array}$ & $\begin{array}{l}\text { EPM } \\
\text { Capoecitab bine x } 1 \text { cycle }\end{array}$ & 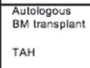 & $\begin{array}{l}\text { Deled(3yoars } \\
\text { after } \\
\text { diagnosis) }\end{array}$ \\
\hline 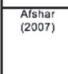 & $\begin{array}{l}331 \\
\text { 619191 } \\
(10011)\end{array}$ & 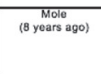 & 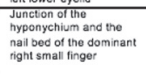 & 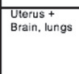 & Chorotocarchoma & $\begin{array}{lll}285.000 \\
\text { milum }\end{array}$ & 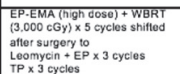 & $\begin{array}{l}\text { TAH } \\
\text { Amputation of } \\
\text { distal phalanx }\end{array}$ & 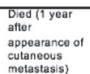 \\
\hline (2004) & & & 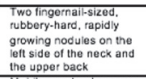 & 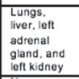 & & $\begin{array}{l}70.000 \\
\text { miưm }\end{array}$ & 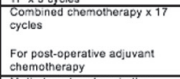 & & \\
\hline $\begin{array}{l}\text { Wolsi } \\
\text { (2001) }\end{array}$ & $\begin{array}{l}310 \\
\text { s190 } \\
(10010)\end{array}$ & $\begin{array}{l}\text { (A Aortlon } \\
\text { (2 years ago) }\end{array}$ & 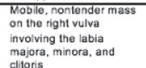 & Nore & Croriocacticioma & $\begin{array}{l}95.000 \\
\text { miUtm }\end{array}$ & 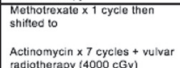 & 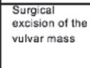 & Remisson \\
\hline $\begin{array}{l}\text { chamama } \\
(2002)\end{array}$ & $\begin{array}{ll}40 \\
6303 \\
(303)\end{array}$ & (15 yearrs ago) & 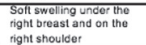 & Lung, weor & Grotococatcinoma & 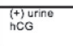 & & None & Alivo \\
\hline $\begin{array}{l}\text { Yruen } \\
\text { Yoge } \\
1996\end{array}$ & $\begin{array}{c}31 \\
6303 \\
(3003)\end{array}$ & $\begin{array}{l}(4 \text { months agoo) } \\
\text { (t) }\end{array}$ & 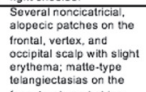 & Lungs & & T15800 & $\begin{array}{l}\text { EMACO } \times 15 \text { cycles } \\
\text { EP } \times 3 \text { cyclos }\end{array}$ & TAH & \\
\hline & & 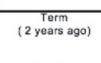 & 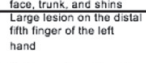 & 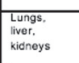 & Noldone & 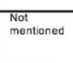 & 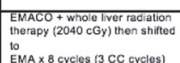 & BTL & Remissior \\
\hline \multirow[t]{2}{*}{ 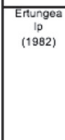 } & 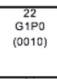 & 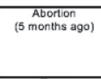 & 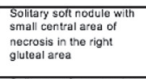 & Lungs & chooricacart & $\begin{array}{llll}632.356 \\
\text { milumb }\end{array}$ & 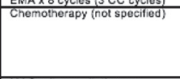 & & 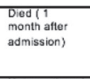 \\
\hline & $\begin{array}{l}31 \\
\text { 6695 } \\
(5015)\end{array}$ & $\begin{array}{ll}\text { (5 } 5 \text { years ago) } \\
\text { Tors }\end{array}$ & 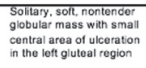 & 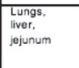 & Croprocatrinoma & $\begin{array}{l}\text { Not } \\
\text { mentioned }\end{array}$ & WAC + IVer racioloterapy & None & Not mentionec \\
\hline $\begin{array}{l}\text { Salimi } \\
(1977)\end{array}$ & & Mole & 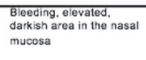 & Lungs & Croofocacterioma & 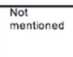 & Nolmentenod & TAHESO & Not mentone \\
\hline $\begin{array}{c}\text { Cosonow } \\
\text { (1974) }\end{array}$ & ${ }^{29}$ & $\begin{array}{l}\text { (3) Aborthon } \\
\text { (3 onths ago) }\end{array}$ & 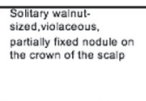 & 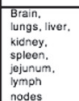 & Crororocartcinoma & 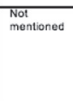 & $\begin{array}{l}\text { Extensive chamolherapy (not } \\
\text { specified) }\end{array}$ & & $\begin{array}{l}\text { Died (3) } \\
\text { months atter } \\
\text { admission) }\end{array}$ \\
\hline
\end{tabular}

